\title{
“EXPERIMENTAL STUDY OF PHOTOELECTRONIC SPECTRA OF NIOBIUM-HAFNIUM-ZIRCONIUM ALLOY"
}

\author{
${ }^{1}$ N.A.Nurmatov, ${ }^{2}$ G.T.Rakhmanov, ${ }^{3}$ D.A.Begmatova, ${ }^{4}$ Sh.Kh.Kamilov, ${ }^{5}$ R.A.Alimov \\ ${ }^{1,2,3,4,5}$ Faculty of Physics, National University of Uzbekistan named after M. Ulugbek, Tashkent, Uzbekistan
}

\begin{abstract}
Crystals of niobium and its alloys obtained by low-energy implantation of hafnium and zirconium ions have been studied in a multifunctional experimental setup. Distribution profiles of implanted hafnium and zirconium atoms, energy distributions of photoelectrons $\mathrm{N}(\mathrm{E})$, and spectral dependences of the quantum yield of photoelectron emission before and before and after heating of the niobium-hafnium-zirconium alloy have been investigated. The contribution of surface states and bands formed by hafnium and zirconium atoms to photoelectron emission in the photon energy range of $8-10 \mathrm{eV}$ is analyzed. The experimental results are discussed on the basis of theoretical calculations of the surface, photoelectron emission in which the dominant factors are indirect optical transitions. The experiments were carried out under ultrahigh vacuum conditions using photoelectron and Auger electron spectroscopy. Krypton and xenon resonance lamps were used as radiation sources.
\end{abstract}

Keywords:

spectrum, photoelectron, alloy, niobium, hafnium, zirconium, experiment, result, energy, ion.

Article Received: 18 October 2020, Revised: 3 November 2020, Accepted: 24 December 2020

\section{INTRODUCTION}

Currently, one of the urgent tasks of physical electronics, microelectronics and nanotechnology is the creation of surface alloys of arbitrary composition with the required concentration of alloying elements. Alloys of refractory metals based on niobium, molybdenum, tantalum and other elements are widely used in nuclear power engineering, aviation technology, mechanical engineering, vacuum technology, etc. Experimental and theoretical studies of a number of alloys have shown that control of the state of $\mathrm{s}$ $-\mathrm{d}$ electrons by introducing low concentrations of alloying elements and creating surface alloys makes it possible to solve a number of fundamental problems of applied and theoretical nature $[1,2]$. Conventional alloys have some disadvantages. One of the disadvantages of such alloys is the difficulty of obtaining atomically pure alloys that do not have a significant amount of carbon atoms, oxygen, sulfur and other impurity elements. Obtaining alloys by implantation of various refractory elements with low-energy ions eliminates the aforementioned disadvantages of alloys. In addition, radiation and other damage to the near-surface region of alloys obtained by this technology insignificantly affects the structure of surface atoms. In this work, we investigate the elemental composition, the change in the spectral dependence of the quantum yield of photoelectron emission QYP, the features of the formation of photoelectron spectra $\mathrm{N}(\mathrm{E})$ in the near and vacuum ultraviolet region for the $\mathrm{Nb}-\mathrm{Hf}-$ $\mathrm{Zr}$ system. The photoemission work function of the multicomponent alloy and the concentration profile of the distribution of zirconium atoms in $\mathrm{Nb}$ (110) are also calculated.

\section{EXPERIMENTAL PROCEDURE}

The design of a multifunctional ultrahigh-vacuum experimental setup with a solid-state ion source and the production of alloys by alloying are described in [1]. The alloy under study was fabricated in the following mode: the doped flux of $\mathrm{Hf}$ and $\mathrm{Zr}$ ions is $\sim 10^{11}$ ion $\mathrm{cm}^{-2} \mathrm{c}^{-1}$, and the ion energy is $\mathrm{E}=3^{-4} \mathrm{keV}$. The working vacuum in the device chamber was (3-5) $10^{-10}$ Torr.

The manipulator of the experimental setup was equipped with hafnium crystals, an $\mathrm{Nb}$ (110) single crystal, and zirconium. The crystals were preheated to a high temperature ( $\mathrm{T}=2200-2400 \mathrm{~K}$ ) for more than 50 hours. The surface cleanliness was monitored using Auger spectra. Auger spectra of the samples were measured in the energy range 
from 80 to $600 \mathrm{eV}$ after high-temperature heating, as well as in heated crystals. Quantitative calculations were performed using the elemental sensitivity factor method. After reaching an atomically clean surface of the samples, the spectral dependences of the CWF were recorded and the value of the work function was determined by the methods of direct and isothermal Fowler curves. In this case $Y^{1 / 2}=f(h v) Y^{1 / 2}=f(h v)$, the dependences and $\mathrm{lg}$ ) were plotted in the photon energy range from $3.95 \mathrm{eV}$ to $5.15 \mathrm{eV}$. Curves of the energy distribution of photoelectrons were obtained at photon energies of $h v=8.4$ and $10 \mathrm{eV}$. Resonant xenon and krypton lamps of the KsR-2-1 and $\mathrm{KrR}-2-1$ types were used as radiation sources. After each thermal cleaning of the samples under study, Auger spectra and spectral dependences of the QYP were recorded from the surface of $\mathrm{Nb}$, $\mathrm{Hf}$, and $\mathrm{Zr}$ crystals in order to compare these results after implantation of niobium with hafnium and zirconium ions.

\section{EXPERIMENTAL RESULTS AND THEIR DISCUSSION}

In fig. 1 shows Auger spectra after implantation of $\mathrm{Hf}$ and $\mathrm{Zr}$ ions into $\mathrm{Nb}$ (110), where the main peaks from niobium, hafnium, and zirconium atoms are seen. The maximum surface concentration of implanted hafnium and zirconium atoms is achieved after heating the sample to 1350-1400 K. Further heating of the crystal (over $1400 \mathrm{~K}$ ) leads to a decrease in the surface concentration of hafnium and zirconium. To find the depth distribution of $\mathrm{Hf}$ and $\mathrm{Zr}$ atoms in $\mathrm{Nb}$ (110), layer-by-layer etching of the crystal surface with argon ions was performed. At the used current density and energy $E p=1 \mathrm{keV}$, one layer of $\mathrm{Hf}$ and $\mathrm{Zr}$ atoms was removed in 7-8 min.
After removal of 8-10 atomic layers from the crystal surface, the concentration of $\mathrm{Hf}$ and $\mathrm{Zr}$ atoms decreased to 1 at. \%. Figure 2 shows the concentration profile of the distribution of ionimplanted zirconium atoms in $\mathrm{Nb}$ (110). It is seen that at a depth of $\sim 3$ atomic layers, the concentration of zirconium reaches $\sim 10-12$ at $\%$. When 8-10 atomic layers are removed, the $\mathrm{Zr}$ concentration decreases to 2-3 atm.\%. According to the existing model of structure formation of dilute ion-implanted metal alloys [3], the structure of a metastable solid solution is formed as a result of rapid cooling of the micro region of a dense collision cascade, in which the processes of collective motion of atoms take place, including the implanted atom. Upon rapid cooling of the cascade region, the crystal structure of the matrix is restored; Based on this model, it can be noted that the position of the doping zirconium atom, which is restored to the lattice of a niobium single crystal, is fixed as a result of the sequential development of a number of processes, including spontaneous recombination of the doping zirconium and hafnium atoms with a mobile vacancy in the niobium lattice. As a result, the zirconium or hafnium atom is located at the niobium lattice site. Thus, at the doses and energy of irradiation with zirconium and hafnium ions we used, a solid solution of substitution of zirconium and hafnium in niobium was formed in the surface layer of niobium up to $\sim 10$ atomic layers thick. The maximum concentration of $\mathrm{Zr}$ is observed at a depth of $\sim 3$ atomic layers from the niobium surface. The change in the concentration of $\mathrm{Hf}$ and $\mathrm{Zr}$ atoms in the surface layer of ion-doped niobium during heating shows that with an increase in the crystal temperature to 1300-1350 $\mathrm{K}$, segregation and thermal diffusion of $\mathrm{Hf}$ and $\mathrm{Zr}$ atoms into the surface layer of the $\mathrm{Nb}-\mathrm{Hf}-\mathrm{Zr}$ system occurs. 


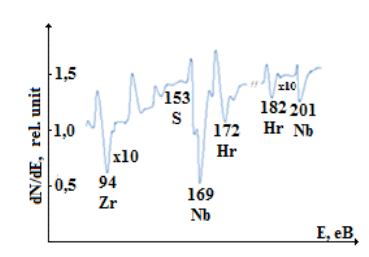

Fig.1. Auger spectrum of alloy Nb-Hf-Zr

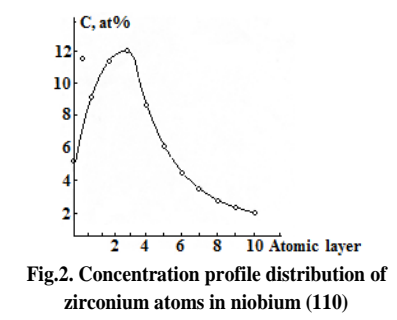

The spectral dependences of the QYP of crystals studied before and after the implantation of the $\mathrm{Nb}$ (110) surface with hafnium and zirconium ions were recorded under the same surface treatment conditions, and the photoemission work function was calculated. If before implantation this value for $\mathrm{Nb}$ (110) is $\mathrm{e} \varphi_{(110)}=4.52 \pm 0.02 \mathrm{eV}$, then after implantation the photoemission work function was $\mathrm{e} \varphi_{(110)}=$ $4.38-4.40 \mathrm{eV}$; the work function decreases by $0.15 \mathrm{eV}$. Next, the $\mathrm{N}$ (E) photoelectron spectra were recorded QYP for the niobium-hafniumzirconium alloy. Calculation of the electronic structure of $\mathrm{Zr}$ and $\mathrm{Nb}$ [7, 9] shows that on the distribution curves of the density of states, two or three peaks are formed due to the contribution mainly of d-electrons and partially s-, p-, and felectrons and their hybridization [9] ... It was shown in [10] that the shape of the localized densities of electronic states of the first two atomic layers corresponds to their bulk density. Thus, when analyzing the experimental $\mathrm{N}$ (E) emission curves from the surface zone, one can use the electronic structure for the bulk of $\mathrm{Nb}, \mathrm{Hf}$, and $\mathrm{Zr}$. The narrowing of the d-band during surface emission can be detected at high photon energies. In order to facilitate the interpretation of the obtained photoelectron spectra $N$ (E) for the $\mathrm{Nb}-\mathrm{Hf}-\mathrm{Zr}$ alloy, the energy distribution curves of photoelectrons were recorded from the surface of $\mathrm{Nb}$ (110), hafnium crystals and an atomically clean zirconium surface. Figure 3 shows the N (E) spectra for a pure crystal of niobium (curve 1) and hafnium (curve 2).

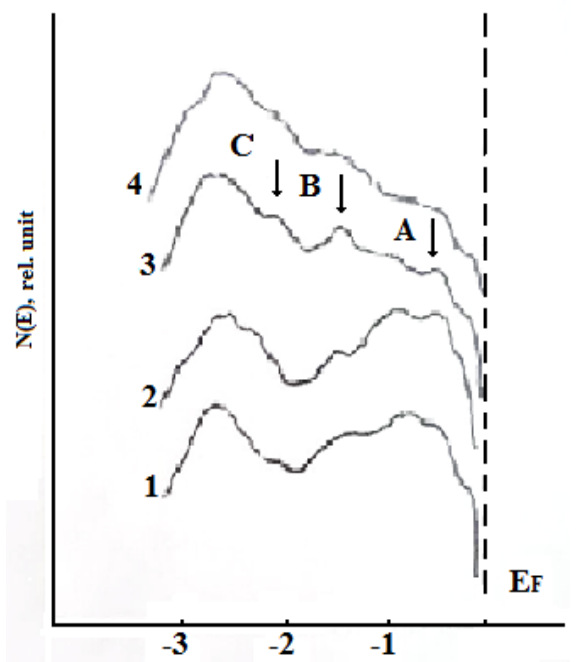

Fig.3. photoelectron spectra of ionic implanted alloy.

1-Nb, 2-Hf, 3-Nb-Hf-Zr after warming up;

T-1400 K, 4-Nb-Hf-Zr after warming up;

T-1200

The same figure shows the $\mathrm{N}$ (E) spectra of the Nb-Hf-Zr alloy (curve 3,4). It is seen that the spectra of photoelectrons recorded from the hafnium surface at a photon energy of $8.4 \mathrm{eV}$ have three maxima at energies of $0.5,1.4,2.4 \mathrm{eV}$ (curve 2). The position of peaks A and B with energies of $-0.5 \mathrm{eV}$ and $-1.4 \mathrm{eV}$ below the Fermi level does not depend on the photon energy. After heating the crystal at $\mathrm{T}=1200 \mathrm{~K}$ (curve 4 ) and $\mathrm{T}$ $=1400 \mathrm{~K}$ (curve 3 ), a number of features are observed in the regions of $0.5 \mathrm{eV}, 1.4-1.5 \mathrm{eV}$, and 2.1-2.2 eV below the Fermi level. The $\mathrm{N}$ (E) curve for $\mathrm{Nb}-\mathrm{Hf}-\mathrm{Zr}$, in contrast to the pure niobium sample, had a wide maximum in the lowenergy region (curve 4), and after heating at $\mathrm{T}=$ $1400 \mathrm{~K}$, a decrease in the intensity of this maximum was observed (curve 3). Comparing these four curves, we can say the following. Peak $\mathrm{B}$ at $1.4-1.5 \mathrm{eV}$ is most likely associated with the density of electronic states of hafnium, since the position of this maximum did not change when the photoelectron spectra were taken at $\mathrm{eV}$. We associate peaks $\mathrm{A}$ and $\mathrm{C}$ with indirect optical transitions [5, 6] for a pure $\mathrm{Nb}$ (110) single crystal. Thus, the photoelectron spectra for pure 
$\mathrm{Nb}$ (110) (curve 1) exhibit maxima due to the band structure of niobium, and in the spectrum obtained after doping with hafnium and zirconium ions an additional maximum appears at $1.4-1.5 \mathrm{eV}$ below the Fermi level ... If, prior to implantation of the $\mathrm{Nb}$ (110) sample, there is a significant increase in the QYP depending on the energy of incident photons, then after implantation, a weak dependence $\mathrm{Y}=\mathrm{f}(h v)$ (is observed. Second, the implanted ions form a substitutional solid solution with the matrix, which leads to a change in the atomic structure and lattice potential. Photoelectrons participating in emission from the surface layer, due to the formation of defects and radiation damage during ion implantation, lose part of their energy. It should be noted that these processes are influenced by the heating of the crystal and the presence of residual gases in the installation. The origin of the low-energy maximum in pure metals is mainly due to the loss of energy of photoelectrons during electronelectron inelastic scattering [3]. With ion doping, a cascade of collisions develops in a solid. Moreover, in the metal In the matrix, a large number of defects such as vacancies and interstitial atoms are formed, which leads to a significant increase in the amplitude of the lowenergy maximum. Note that our data for a clean $\mathrm{Nb}$ (110) surface are in good agreement with the results [7]. The positions of peaks $\mathrm{A}$ and $\mathrm{B}$ with energies 0.5 and $1.4 \mathrm{eV}$ below the Fermi level do not depend on the photon energy. Such features for the $N$ (E) curves in the model of indirect transitions [3] correspond to the maxima of the density of electronic states of the valence band. It has been experimentally established that the peak located immediately near the Fermi level $(\sim 0.5$ $\mathrm{eV}$ ) is very sensitive to surface contamination by adsorbed gases. Most likely, this peak is associated with the surface resonance level. An analysis of the $\mathrm{N}$ (E) curves recorded at photon energies of 10 and $8.4 \mathrm{eV}$ from the surface of iondoped niobium shows that additional maxima at energies $1.4-1.5 \mathrm{eV}$ below the Fermi level are due to implanted hafnium and zirconium atoms $[6,7]$.
The implanted hafnium and zirconium atoms create their own energy sub bands associated with the d-electrons of hafnium and zirconium. Most likely, these electronic sub bands are located in the forbidden band in the direction of symmetry of the $\Gamma \mathrm{N}$ band structure of the $\mathrm{Nb}$ (110) single crystal $[5,6]$. Analyzing the results obtained, we note that model calculations of the yield of electrons from bulk and surface states, carried out by a number of authors $[1,6]$, showed that the contribution of surface states is made from $\sim 2.5$ atomic layers.

\section{CONCLUSION}

Thus, an analysis of the experimental curves $\mathrm{N}$ (E), spectral distributions of the QYP, the distribution of elements in depth and electron Auger spectra, as well as comparison with calculations of their band structure shows: 1) Low-energy ion implantation of hafnium and zirconium atoms in niobium noticeably changes the shape of the distribution QYP and N (E) for both pure niobium, zirconium and hafnium. 2) Additional maxima associated with d electrons of hafnium and zirconium atoms appear in the photoelectron spectra; 3) Near the Fermi level, surface states are formed that are very sensitive to the influence of residual gases in the experimental setup. These surface states are also manifested in the spectral dependence of the QYP.

\section{REFERENCES}

[1] Buribaev, N.A. Nurmatov, Y.S. Ergashov, N. Tolipov, A. Kholov. "Investigation of the photoelectron spectra of an ion-implanted single crystal of Mo (111)" // Surface. Xray., Synchrotron. and a neutron. issled. 2012. №. 9. pp. 55-58.

[2] Senkovsky B.V., Usachev D.Yu., Fedorov A.V., Adamchuk V.K. "Experimental study of the valence band of $\mathrm{Ti}(\mathrm{Ni} \mathrm{Cu})$ alloys with different compositions and crystal structures", FTT, 2012, v.54, issue 8

[3] Buribaev I., Nurmatov NA, "Contribution of surface states to the photoelectron emission 
of zirconium and niobium". // Surface. X-

ray, synchrometer. and a neutron. Issled.2001, №. 6., pp. 99-103.

[4] Cinti R.S., Khoury E.Al., Chakraverty B.K., Christensen N.E. "Directional UV photoemission from (100) and (110) molybdenum surfaces" // Phys. Rev. B. 1976 V. 14. № 8. pp. 3296.

[5] Buribaev I., Nurmatov N.A." UV photoelectron spectra of $\mathrm{Nb}$ and Mo singlecrustal surfaces" // Journ. Of Electron. Spectrosc. Relat. Phenom. 1994. V. 68. pp. 547-554

[6] Ehrenreich G., Schwartz L. // Electronic structure of alloys. Moscow: Mir, 1979. pp. 200.

[7] Jepsen O., Andersen O.K., Mackintosh A.R. Electronic structure of hcp transition metals// Phys. Rev. B. - 1975, - V.12. - № 8. - pp. 3084-3103.

[8] Buribaev I., Nurmatov N.A, Talipov N. The experimental studies on $\mathrm{Zr}$ Photoelectron Spectra. // Turkish Journal of Physics. 1999.-v.23.-№ 3.-pp. 463-470.

[9] Elyasher N., Koelling D.D. Self- consisten relativistic APW calculation of the electronic structure of niobium with a nonmiffintin potential. Phys. Rev. B. - 1977, -V 15, - №8, - pp. 3620-3632.

[10] Buribaev I., Nurmatov N.A., Ergashov Y.S., Tolipov N., Kholov A. "Investigation of the photoelectric spectra of an ion-implanted Mo (111) single crystal" // J. Surface. X-ray, synchrotron and neutron research. -2012. №. 9 pp. 55-58. 